\title{
Effect of Nursing Care Guidelines for Post Anesthetic (Phase One) on Patient Outcome at Assiut University Hospital
}

\author{
Marzoq Ali Odhah, Hany Ahmed Ibrahim, Mona Aly Mohammed \& Mervat Anwar AbdEl-Aziz \\ Clinical Nurse Specialist in Kuwait University Hospital Sana'a,Yemen. \\ Professor of Anesthesia, Faculty, of Medicine, Assiut University, Egypt. \\ Lecturer of critical care Nursing, Faculty of Nursing, Assiut University, Egypt. \\ Lecturer of critical care Nursing, Faculty of Nursing, Assiut University, Egypt.
}

\begin{abstract}
Background: The post anesthesia nurse assumes important for the care and clinical stabilization of the patient in the immediate postanesthetic phase one period until they are fit. Aim this study was carried out to investigate the effect of nursing care guidelines for postanesthetic (phase one) on patient outcome. Tools three tools were utilized to collect data pertinent to the study, which is Tool I Patients assessment sheet consists of three parts. Tool II Richmond Agitation Sedation Scale. Tool III faces pain scales. Methods a quasi-experimental design. The sample was (102) patients that divided into two groups (control 51 and study 51) collected over sixth months. Results As regarding to all time intervals hemodynamic parameters, respiratory status and immediately post anesthesia pain related operation there were statistically significant difference $(\mathrm{p}<001)$ between control and study groups post anesthesia phase one, also As regard post-anesthesia complication reduce with statistically significant difference relation to airway obstruction, pain related operation and hypothermia $(\mathrm{p}<0.009, \mathrm{p}=0.029$ and $\mathrm{p}=0.017)$ respectively between both groups. Conclusion apply nursing care guideline for post-anesthetic showed statistically significance difference of hemodynamic parameters, respiratory status outcomes, decrease pain level and complication.
\end{abstract}

\section{Keywords: Post Anesthesia Phase one, Nursing Guideline \& Patient Outcome.}

\section{Introduction}

Phase I postanesthesia care involves providing intensive monitoring and care of the patient in the immediate postoperative period, Patients move from Phase I care either to Phase II care in the PACU or to an inpatient unit. Phase II postanesthesia care provides less intensive care to surgery patients who will then be transferred to Phase III care, to home, or to an extended care facility. Phase III postanesthesia care is provided when patients are being prepared for self-care and discharge from the surgical facility(Davis et al., 2012).

Post-anesthesia care unit is a complex process related to various outcomes, such as physiological endpoints, the incidence of adverse events, and change in psychological status. Previous studies of recovery after surgery and anesthesia have focused primarily on the physiological end-points, and the incidence of adverse events, including major morbidity and mortality. However, because of the advances in both surgical and anesthetic techniques, particularly in ambulatory surgery, mortality and major morbidity have become rare event (Silverstein, et al., 2013). Immediately after the surgical procedure is complete, the client is transported to the postanesthesia care unit, also known as the postanesthesia recovery room, located near the operation room. The nursing staff there is specifically knowledgeable in the care of clients recovering from anesthesia. Specialized equipment is available to monitor and treat the client. Surgical and anesthesia personnel is immediately available for any emergencies (Timby, \& Smith, 2010).

Nursing guidelines for phase I focused on $A B C$ : airway, breathing, and circulation. Monitor the patient's airway, gas exchange, pulse oximetry, oxygen delivery, accessory muscle use, and breathe sounds. The cardiovascular status is checked next. Vital signs are checked every 15minutes until stabilized; pulse, blood pressure, and cardiac rhythm are monitored, check the level of consciousness, level of pain and is monitored for the presence of nausea or vomiting (Digiulio et al., 2007).

\footnotetext{
Aim of the Study

To investigate the effect of nursing care guidelines for postanesthetic (phase one) on patient's outcome at Assiut University Hospital
} 


\section{Significance of the study}

The significance of the study the patients post anesthesia needed a special nursing care to improve the patient outcome and prevention complication. So this study improved the nursing care through applied nursing care guideline that was the baseline for quality nursing care and skills that are needed to practice safely for such a group of patients.

\section{Patients \& Methods}

\section{Research design}

The present study used quasi-experimental research design. This design allowed the research to control the time when the subject is observed and exposed to the procedure.

\section{Research hypotheses}

- A significant difference between hemodynamic parameters with phase one time post anesthesia (30, 60, 90, 120min).

- Significant difference between pains levels with difference phase one time post anesthesia $(30,60$, 90, 120min).

- A significant difference between respiratory monitoring parameters with phase one time post anesthesia $(30,60,90,120 \mathrm{~min})$.

\section{Operational definition}

Nursing care guideline: 'are systematically developed recommendations that assist the practitioner and patient in making decisions about health care.(Silverstein et al., 2013)

Phase I: is the initial phase, involving the first two hours, is considered to be the acute phase. The subsequent period of recovery (in hospital) (Lethbridge, 2008)

Outcome: the outcome is defined as "a change in a patient's health status that can be attributed to antecedent health care." Improved patient outcome after surgery is one of the ultimate goals of any anesthetic technique.(van Beest et al., 2014)

\section{Setting of the study}

Data were conducted in microsurgical reconstructive post anesthesia care unit at Assiut University Hospital.

\section{Sampling}

Data were collected over sixth months (from December 2013 to May 2014) and the sample was (102) patients that divided into two groups (control 51 patients and study 51 patients) post-anesthetic (phase one) in microsurgical reconstructive post anesthesia care unit.

\section{Inclusion criteria}

- All patient post-anesthetic (phase one) in the microsurgical reconstructive intensive care unit.

- Adult patient age from 18-60 years

- Immediately two hours post anesthesia

Exclusion criteria

Patients refuse participation in this study

Tools of the study

Three tools were used to collect the data in this study and developed by the researcher based on the review of relating literature (Smedley, \& Goldworthy, 2012, Tennant, et al., 2012).

Tool I: Patients assessment sheet consists of three parts

Part (1): preoperative assessment

- Socio-demographic data of the patient as code, age, sex

Part (2): intraoperative assessment

- Clinical data as type of anesthesia and duration of anesthesia

Part (3): immediately post-anesthesia assessment sheet

(Nursing care guidelines)

\section{- Hemodynamic parameters}

The baseline for blood pressure: mean arterial pressure, heart rate, and rhythm, temperature, Nail beds for a capillary refill, Peripheral arterial pulses for rhythm, bilateral equality where indicated, ECG monitoring every 15 minutes for two hours

\section{- Respiratory Assessment}

Adequacy of airway, respiratory effort [rate, depth, pattern of breathing, use of accessory muscles], the level of oxygenation - saturation of oxygen measured by pulse oximetry, auscultation for breath sounds, every 15 minutes for two hours.

\section{Immediately postoperative complication}

This tool was used to assess the frequency and percent of the complications relevant post anesthesia. Includes hypotension, bradycardia, hypertension, tachycardia, airway obstruction, hypoxia, laryngeal spasm, nausea, vomiting, headache, myalgia, oral trauma, teeth trauma, back pain, hypothermia, hyperthermia, shivering. 


\section{Tool II: Richmond Agitation-Sedation Scale} (Sessler, et al., 2003)

It is a ten point scale with levels for assessing anxiety and agitation, one for an alert and calm state and further levels for quality of sedation. Three sequential steps are used: observation, response to verbal stimulation and response to physical stimulation. We measured interrater reliability and validity of a new 10-level (+4 "combative" to -5 "unarousable") scale. The researcher will adopt.

Tool III: faces pain scales: (Warden, et al., 2011) Total scores range from 0 to 10 (based on a scale of 0 to 2 for five items), with a higher score indicating

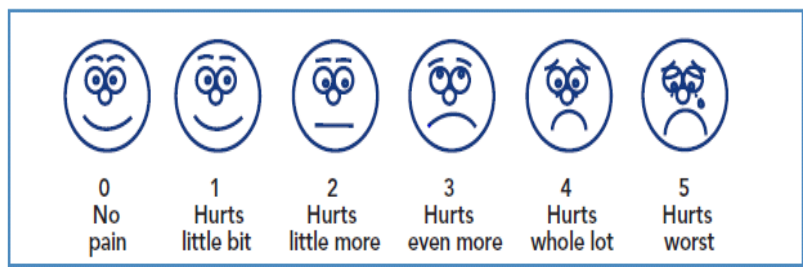

more severe pain ( $0="$ no pain" to $10="$ severe pain").

\section{Methods}

- Secure necessary approval, a written letter from the Faculty of Nursing will be submitted to the directors of the study settings to get permissions for data collection.

- An official permission to conduct the study was obtained from hospital responsible authorities in the anesthesiology department and microsurgical reconstructive operation after explaining the aim and nature of the study.

- Ethical consideration the nature and purpose of the study were explained to every patient.

- Informed consent was obtained from every relative of the patient.

- The anonymity and confidentiality of responses, voluntary participation and the right to refuse to participate in the study were emphasized to subject.

- The tools used in this study were developed by the researcher based on reviewing the relevant literature

- Content validity: the tools were reviewed and tested by jury for face the feasibility, applicability and the content validity of the tools and all the necessary modification done. The jury members were five experts represent related content. This included specialists in the field three specialists of critical care nursing and two specialists critical care medicine from Assiut University and the necessary modification done.

- A pilot study was conducted on five patients postanesthetic (phase one) admitted to microsurgical reconstructive intensive care unit to test tools. These selected patients don't include in the main study sample. The necessary modification was done prior to data collection, to test the reliability analysis test using Alpha Cronbach's values (0.943) and applicability of tools.

- The studied sample fulfilling the research criteria was assigned to two groups (the control group and the study group).

- The control group received usual care of the hospital without intervention from the researcher.

- The study group received post anesthesia nursing care guidelines.

\section{Research implementation (procedure)}

This study was carried out in three phases

\section{Phase I: preparatory phase}

A reviewer of current and past, local and international related literature in the various aspects using books, articles, and periodicals, the magazine was done. The proposed study setting was assessed for a number of patients admitted to microsurgical reconstructive post anesthesia care unit, this ended by a pilot study.

\section{Phase II: implementation phase}

- The control group received habitual care of the hospital without intervention from the researcher. Data were collected in post-anesthetic (phase one) microsurgical reconstructive post anesthesia care unit at Assiut University Hospital during December 2013 -May 2014, the tools were filed through interviewing patients during 30 minutes before anesthesia. Implementation nursing care guidelines through two hours immediately post anesthesia for the study group. The study was carried out during the morning for all patients available in microsurgical reconstructive post anesthesia care unit.

- Interview the select patient to fill assessment sheet for part of socio-demographic data as code, age, and sex by researcher using tool I part one before half hour of operation

- Observe patient during the intraoperative period for recorded of type anesthesia, and duration of 
anesthesia by the researcher using the tool I part two.

\section{Implementation nursing care guidelines}

The nursing care guideline implementation by researcher using tools I, II \& III immediately post anesthesia monitoring every 15 minutes for two hours by immediately post-anesthesia assessment sheet that include:

- Hemodynamic parameters and respiratory assessment every 15 minutes for two hours by the researcher using the tool I part three.

- Richmond Agitation Sedation Scale (RASS):

- Three sequential steps are used: observation, response to verbal stimulation and response to physical stimulation by the researcher using tool II every 15 minutes for two hours.

- Faces pain scale

This scale for assessment pain level by the facial expression for post anesthesia patient by researcher used tool III every 15 minutes for two hours.

Phase III: evaluation phase

Both control and study groups were evaluated during post-anesthesia phase one using (tools I, II \& III) for the patient to determine the effect of implementing nursing care guideline to the study group.

\section{Results}

Table (1): comparison between control and study groups of patients according to socio-demographic characteristics, types and duration of anesthesia (No. 102).

\begin{tabular}{|c|c|c|c|c|c|}
\hline \multirow{2}{*}{ Items } & \multicolumn{2}{|c|}{ Control $(n=51)$} & \multicolumn{2}{|c|}{ Study $(n=51)$} & \multirow{2}{*}{ P. value } \\
\hline & No. & $\%$ & No. & $\%$ & \\
\hline \multicolumn{5}{|l|}{ Age group } & \multirow{4}{*}{$0.218^{\mathrm{Ns}}$} \\
\hline 18- 30 years & 30 & 58.8 & 25 & 49.0 & \\
\hline$>30-45$ years & 14 & 27.5 & 22 & 43.1 & \\
\hline$>45-60$ years & 7 & 13.7 & 4 & 7.8 & \\
\hline Mean \pm SD & \multicolumn{2}{|c|}{$31.5 \pm 11.8$} & \multicolumn{2}{|c|}{$31.9 \pm 9.2$} & 0.852 \\
\hline \multicolumn{5}{|l|}{ Gander } & \multirow{3}{*}{$0.141^{\mathrm{Ns}}$} \\
\hline Male & 37 & 72.5 & 44 & 86.3 & \\
\hline Female & 14 & 27.5 & 7 & 13.7 & \\
\hline \multicolumn{5}{|l|}{ Types of anesthesia } & \multirow{4}{*}{$0.799^{\mathrm{Ns}}$} \\
\hline General anesthesia & 46 & 90.2 & 46 & 90.2 & \\
\hline Spinal/epidural anesthesia & 4 & 7.8 & 4 & 7.8 & \\
\hline Local anesthesia & 1 & 2.0 & 1 & 2.0 & \\
\hline \multicolumn{5}{|l|}{ Duration of anesthesia } & \multirow{4}{*}{$0.154^{\mathrm{Ns}}$} \\
\hline $1-2 \mathrm{hr}$ & 14 & 27.5 & 21 & 41.2 & \\
\hline$>2-4 \mathrm{hr}$. & 29 & 56.9 & 27 & 52.9 & \\
\hline$>4 \mathrm{hr}$. & 8 & 15.7 & 3 & 5.9 & \\
\hline Mean \pm SD & \multicolumn{2}{|c|}{$3.2 \pm 1.2$} & \multicolumn{2}{|c|}{$2.8 \pm 1.0$} & $0.101^{\mathrm{Ns}}$ \\
\hline Total & 51 & 100.0 & 51 & 100.0 & \\
\hline
\end{tabular}

Chi-square test was used to compare percentages and independent t-test was used to compare means

${ }^{N s}$ : There is no significant difference $(p>0.05)$ 
Table (2): comparison between control and study groups patient according to Richmond Agitation Sedation Scale (RASS) (No. 102).

\begin{tabular}{|c|c|c|c|c|c|c|c|c|c|c|c|c|c|c|c|c|}
\hline \multirow{4}{*}{ Item } & \multicolumn{16}{|c|}{ Richmond Agitation Sedation Scale (RASS) } \\
\hline & \multicolumn{4}{|c|}{$30 \mathrm{~min}$} & \multicolumn{4}{|c|}{$60 \mathrm{~min}$} & \multicolumn{4}{|c|}{$90 \mathrm{~min}$} & \multicolumn{4}{|c|}{$120 \mathrm{~min}$} \\
\hline & \multicolumn{2}{|c|}{$\begin{array}{c}\text { Control } \\
(n=51)\end{array}$} & \multicolumn{2}{|c|}{$\begin{array}{c}\text { Study } \\
(n=51)\end{array}$} & \multicolumn{2}{|c|}{$\begin{array}{c}\text { Control } \\
(n=51) \\
\end{array}$} & \multicolumn{2}{|c|}{$\begin{array}{c}\text { Study } \\
(n=51)\end{array}$} & \multicolumn{2}{|c|}{$\begin{array}{c}\text { Control } \\
(n=51)\end{array}$} & \multicolumn{2}{|c|}{$\begin{array}{c}\text { Study } \\
(n=51)\end{array}$} & \multicolumn{2}{|c|}{$\begin{array}{c}\text { Control } \\
(n=51) \\
\end{array}$} & \multicolumn{2}{|c|}{$\begin{array}{c}\text { Study } \\
(n=51)\end{array}$} \\
\hline & No. & $\%$ & No. & $\%$ & No. & $\%$ & No. & $\%$ & No. & $\%$ & No. & $\%$ & No. & $\%$ & No. & $\%$ \\
\hline $\begin{array}{l}\text { Very agitated } \\
(+3)\end{array}$ & 2 & 3.9 & 0 & 0.0 & 0 & 0.0 & 0 & 0.0 & 0 & 0.0 & 0 & 0.0 & 0 & 0.0 & 0 & 0.0 \\
\hline Agitated (+2) & 7 & 13.7 & 1 & 2.0 & 0 & 0.0 & 0 & 0.0 & 0 & 0.0 & 0 & 0.0 & 0 & 0.0 & 0 & 0.0 \\
\hline Restless (+1) & 11 & 21.6 & 16 & 31.4 & 7 & 13.7 & 1 & 2.0 & 3 & 5.9 & 0 & 0.0 & 0 & 0.0 & 0 & 0.0 \\
\hline $\begin{array}{l}\text { Alert and } \\
\text { calm (0) }\end{array}$ & 8 & 15.7 & 16 & 31.4 & 37 & 72.5 & 50 & 98.0 & 45 & 88.2 & 51 & 100.0 & 50 & 98.0 & 51 & 100.0 \\
\hline $\begin{array}{l}\text { Drowsy } \\
(-1)\end{array}$ & 16 & 31.4 & 18 & 35.3 & 7 & 13.7 & 0 & 0.0 & 3 & 5.9 & 0 & 0.0 & 1 & 2.0 & 0 & 0.0 \\
\hline $\begin{array}{l}\text { Light sedation } \\
(-2)\end{array}$ & 7 & 13.7 & 0 & 0.0 & 0 & 0.0 & 0 & 0.0 & 0 & 0.0 & 0 & 0.0 & 0 & 0.0 & 0 & 0.0 \\
\hline P. Value & & 0.00 & & & & 0.0 & & & & & $41 *$ & & & & $15^{\mathrm{Ns}}$ & \\
\hline
\end{tabular}

Chi-square test was used to compare percentages.

Ns: There is no significant difference $(P>0.05)$

** There is a significant difference $(P<0.01)$

0 only one answer for each ite.

Table(3): Comparison between control and study patients according to post-operative complications (No. 102).

\begin{tabular}{|c|c|c|c|c|c|}
\hline \multirow{2}{*}{ Complication } & \multicolumn{2}{|c|}{ Control $(n=51)$} & \multicolumn{2}{|c|}{ Study $(n=51)$} & \multirow{2}{*}{ P. value } \\
\hline & No. & $\%$ & No. & $\%$ & \\
\hline \multicolumn{6}{|l|}{ Cardiovascular } \\
\hline Bradycardia & 8 & 15.7 & 7 & 13.7 & $0.999^{\mathrm{Ns}}$ \\
\hline Hypotension & 15 & 29.4 & 8 & 15.7 & $0.155^{\mathrm{Ns}}$ \\
\hline Tachycardia & 12 & 23.5 & 10 & 19.6 & $0.809^{\mathrm{Ns}}$ \\
\hline Hypertension & 6 & 11.8 & 3 & 5.9 & $0.485^{\mathrm{Ns}}$ \\
\hline \multicolumn{6}{|l|}{ Respiratory } \\
\hline Airway obstruction & 8 & 15.7 & 0 & 0.0 & $0.009 * *$ \\
\hline Hypoxia & 9 & 17.6 & 4 & 7.8 & $0.234^{\mathrm{Ns}}$ \\
\hline Laryngeal spasm & 8 & 15.7 & 9 & 17.6 & $0.999^{\mathrm{Ns}}$ \\
\hline \multicolumn{6}{|l|}{ Gastric } \\
\hline Nausea & 12 & 23.5 & 8 & 15.6 & $0.824^{\mathrm{Ns}}$ \\
\hline Vomiting & 12 & 23.5 & 8 & 15.6 & $0.999^{\mathrm{Ns}}$ \\
\hline \multicolumn{6}{|l|}{ Nerves } \\
\hline Back pain & 3 & 5.9 & 1 & 2.0 & $0.609^{\mathrm{Ns}}$ \\
\hline Pain related operation & 31 & 60.8 & 19 & 37.3 & $0.029 *$ \\
\hline Hypothermia & 29 & 56.9 & 16 & 31.4 & $0.017^{*}$ \\
\hline Shivering & 17 & 33.3 & 11 & 21.6 & $0.267^{\mathrm{Ns}}$ \\
\hline
\end{tabular}

Chi-square test was used to compare percentages

** There is a significant difference $(p<0.01)$

NA: not applicable
* There is a significant difference $(p<0.05)$

$N s$ : There is no significant difference $(p>0.05)$

Hypothesis (1): Significant difference between hemodynamic parameters with phase one post anesthesia (30, 60, 90, $120 \mathrm{~min})$ 
Table (4): comparison between control and study groups patient according to hemodynamic parameters

\begin{tabular}{|c|c|c|c|c|c|c|c|c|c|c|c|c|c|c|}
\hline \multirow{3}{*}{$\begin{array}{c}\text { Homodynamic } \\
\text { parameters }\end{array}$} & \multicolumn{2}{|c|}{ Baseline } & \multicolumn{2}{|c|}{$30 \mathrm{~min}$} & \multirow{3}{*}{$\begin{array}{c}\text { P. } \\
\text { value }\end{array}$} & \multicolumn{2}{|c|}{$60 \mathrm{~min}$} & \multirow{3}{*}{$\begin{array}{c}P . \\
\text { value }\end{array}$} & \multicolumn{2}{|c|}{$90 \mathrm{~min}$} & \multirow{3}{*}{$\begin{array}{c}P . \\
\text { value }\end{array}$} & \multicolumn{2}{|c|}{$120 \mathrm{~min}$} & \multirow{3}{*}{$\begin{array}{c}P . \\
\text { value }\end{array}$} \\
\hline & $\begin{array}{l}\text { Control } \\
(\mathrm{n}=51)\end{array}$ & $\begin{array}{l}\text { Study } \\
(\mathrm{n}=51)\end{array}$ & $\begin{array}{l}\text { Control } \\
(\mathrm{n}=51)\end{array}$ & $\begin{array}{l}\text { Study } \\
(\mathrm{n}=51)\end{array}$ & & $\begin{array}{l}\text { Control } \\
(\mathrm{n}=51)\end{array}$ & $\begin{array}{l}\text { Study } \\
(n=51)\end{array}$ & & $\begin{array}{l}\text { Control } \\
(\mathrm{n}=51)\end{array}$ & $\begin{array}{l}\text { Study } \\
(\mathrm{n}=51)\end{array}$ & & $\begin{array}{c}\text { Control } \\
(\mathrm{n}=51)\end{array}$ & $\begin{array}{l}\text { Study } \\
(\mathrm{n}=51)\end{array}$ & \\
\hline & $\underset{\text { SD }}{\text { Mean } \pm}$ & $\underset{\text { SD }}{\text { Mean } \pm}$ & $\underset{\text { SD }}{\text { Mean } \pm}$ & Mean \pm SD & & $\underset{\text { SD }}{\text { Mean } \pm}$ & $\underset{\text { SD }}{\text { Mean } \pm}$ & & $\begin{array}{c}\text { Mean }+ \\
\text { SD }\end{array}$ & $\begin{array}{c}\text { Mean }+ \\
\text { SD }\end{array}$ & & $\begin{array}{c}\text { Mean }+ \\
\text { SD }\end{array}$ & $\begin{array}{c}\text { Mean }+ \\
\text { SD }\end{array}$ & \\
\hline Temperature $\left({ }^{\circ} \mathrm{C}\right)$ & $35.8+0.6$ & $36.1 \pm 0.5$ & $35.8 \pm 0.6$ & $36.9+0.3$ & $0.001 * *$ & $36.3+0.6$ & $37.4 \pm 0.2$ & $0.001 * *$ & $36.5+0.6$ & $37.3 \pm 0.2$ & $0.001^{* *}$ & $36.6 \pm 0.6$ & $37.3+0.2$ & $0.001^{\text {** }}$ \\
\hline $\begin{array}{l}\text { Systolic blood } \\
\text { pressure (mmHg) }\end{array}$ & $110.5 \pm 22.3$ & $109.7 \pm 18.3$ & $113.1 \pm 21.4$ & $119.5+11.9$ & $0.066^{\mathrm{Ns}}$ & $114.5 \pm 17.1$ & $122.2 \pm 7.4$ & $0.004 * *$ & $116 \pm 15.5$ & $123.3 \pm 6.5$ & $0.002 * *$ & $117.1 \pm 15.1$ & $122.4 \pm 6.8$ & $0.024 *$ \\
\hline $\begin{array}{c}\text { Diastolic blood } \\
\text { pressure (mmHg) }\end{array}$ & $59.3 \pm 18.8$ & $58.8 \pm 16.7$ & $60.7 \pm 17.9$ & $66.2 \pm 10.4$ & $0.064^{\mathrm{Ns}}$ & $64.7 \pm 12.2$ & $70.1 \pm 7.1$ & $0.008 * *$ & $65.7 \pm 12.2$ & $71.9 \pm 7$ & $0.002 * \%$ & $65.6 \pm 12.7$ & $72 \pm 5.8$ & $0.001^{* *}$ \\
\hline $\begin{array}{l}\text { Mean arterial blood } \\
\text { pressure }(\mathrm{mmHg})\end{array}$ & $75.2 \pm 21.1$ & $76.5 \pm 16.3$ & $82.4 \pm 22$ & $75.5 \pm 7.2$ & $0.035^{*}$ & $80.4 \pm 14.4$ & $87.4 \pm 6.2$ & $0.002 * *$ & $81.6 \pm 14.1$ & $88.8+6.2$ & 0.001 ** & $81.6 \pm 12.5$ & $88.4+5.7$ & $0.001^{* *}$ \\
\hline Heart rate $(\mathbf{b} / \mathbf{m})$ & $91 \pm 23.7$ & $88.3 \pm 18.4$ & $91.8 \pm 20.6$ & $80.3+9.6$ & $0.001 * *$ & $85.5+18.9$ & $79.5+4.6$ & $0.029 *$ & $84.2 \pm 17.7$ & $78.5+4.8$ & $0.028 *$ & $85 \pm 15.9$ & $80 \pm 4.7$ & $0.035^{*}$ \\
\hline Capillary refill & $2.4 \pm 0.3$ & $2 \pm 0.3$ & $2.3 \pm 0.3$ & $1.7 \pm 0.2$ & $0.001 * *$ & $2.1 \pm 0.3$ & $1.4 \pm 0.2$ & $0.001 * *$ & $2 \pm 0.3$ & $1.4 \pm 0.2$ & $0.001^{* *}$ & $1.9 \pm 0.3$ & $1.3 \pm 0.2$ & $0.001^{* *}$ \\
\hline \multicolumn{14}{|l|}{ ECG monitoring } & \multirow{4}{*}{$0.001^{* *}$} \\
\hline Normal & $23(45.1 \%)$ & $33(64.7 \%)$ & $27(52.9 \%)$ & $48(94.1 \%)$ & \multirow{3}{*}{$0.001 * *$} & $29(56.9 \%)$ & $51(100 \%)$ & \multirow{3}{*}{$0.001 * *$} & $31(60.8 \%)$ & $51(100 \%)$ & \multirow{3}{*}{$0.001^{* *}$} & $34(66.7 \%)$ & $51(100 \%)$ & \\
\hline Bradycardia & $8(15.7 \%)$ & $4(7.8 \%)$ & $8(15.7 \%)$ & $1(2 \%)$ & & $8(15.7 \%)$ & $0(0 \%)$ & & $8(15.7 \%)$ & $0(0 \%)$ & & $7(13.7 \%)$ & $0(0 \%)$ & \\
\hline Tachycardia & $20(39.2 \%)$ & $14(27.5 \%)$ & $16(31.4 \%)$ & $2(3.9 \%)$ & & $14(27.5 \%)$ & $0(0 \%)$ & & $12(23.5 \%)$ & $0(0 \%)$ & & $10(19.6 \%)$ & $0(0 \%)$ & \\
\hline
\end{tabular}

Immediately post anesthesia phase one (No. 102). Chi-square test was used to compare percentages and independent $t$-test was used to compare means

* There is a significant difference $(P<0.05)$

** There is a significant difference $(P<0.01)$

${ }^{N s}$ : There is no significant difference $(P>0.05)$

Baseline is first reading immediately post anesthesia

Hypothesis (2): Significant difference between pain level with difference phase one post anesthesia (30, 60, 90, 120min)

Table (5): comparison between control and study groups patient according to face pain scale immediately post anesthesia (No. 102).

\begin{tabular}{|c|c|c|c|c|c|c|c|c|c|c|c|c|c|c|}
\hline \multirow{3}{*}{ Items } & \multicolumn{2}{|c|}{ Baseline } & \multicolumn{2}{|c|}{$30 \mathrm{~min}$} & \multirow{3}{*}{ P. value } & \multicolumn{2}{|c|}{$60 \mathrm{~min}$} & \multirow{3}{*}{ P. value } & \multicolumn{2}{|c|}{$90 \mathrm{~min}$} & \multirow{3}{*}{$\begin{array}{c}\text { P. } \\
\text { value }\end{array}$} & \multicolumn{2}{|c|}{$120 \mathrm{~min}$} & \multirow{3}{*}{ P. value } \\
\hline & $\begin{array}{c}\text { Control } \\
(\mathrm{n}=51)\end{array}$ & $\begin{array}{c}\text { Study } \\
(\mathrm{n}=51)\end{array}$ & $\begin{array}{c}\text { Control } \\
(\mathrm{n}=51)\end{array}$ & $\begin{array}{l}\text { Study } \\
(\mathrm{n}=51)\end{array}$ & & $\begin{array}{c}\text { Control } \\
(\mathrm{n}=51)\end{array}$ & $\begin{array}{c}\text { Study } \\
(\mathrm{n}=51)\end{array}$ & & $\begin{array}{c}\text { Control } \\
(\mathrm{n}=51)\end{array}$ & $\begin{array}{l}\text { Study } \\
(\mathrm{n}=51)\end{array}$ & & $\begin{array}{c}\text { Control } \\
(\mathrm{n}=51)\end{array}$ & $\begin{array}{l}\text { Study } \\
(\mathrm{n}=51)\end{array}$ & \\
\hline & $\begin{array}{c}\text { Mean } \\
\text { D }\end{array}$ & $\begin{array}{c}\text { Mean } \pm S \\
\text { D }\end{array}$ & $\begin{array}{c}\text { Mean } \pm S \\
\text { D }\end{array}$ & $\begin{array}{c}\text { Mean } \pm S \\
\text { D }\end{array}$ & & $\begin{array}{c}\text { Meant } \\
\text { SD }\end{array}$ & $\begin{array}{c}\text { Meant } \\
\text { SD }\end{array}$ & & $\begin{array}{c}\text { Mean } \pm S \\
\text { D }\end{array}$ & Mean \pm SD & & $\mathrm{Mean}_{ \pm} \mathrm{SD}$ & $\begin{array}{c}\text { Meant } \\
\text { SD }\end{array}$ & \\
\hline Pain & $5.7 \pm 3.1$ & $3.8 \pm 2.6$ & $5.8 \pm 2.6$ & $1.2 \pm 1.1$ & $0.001 * *$ & $5.3 \pm 2.5$ & $0.2 \pm 0.5$ & $0.001 * *$ & $5.2 \pm 2.4$ & $0.1 \pm 0.5$ & $\begin{array}{c}0.001 \\
* *\end{array}$ & $5 \pm 2.3$ & $0 \pm 0$ & $0.001 * *$ \\
\hline
\end{tabular}

Chi-square test was used to compare percentages and independent t-test was used to compare means

* There is a significant difference $(P<0.05)$

There is a significant difference $(P<0.01)$

${ }^{N s}$ : There is no significant difference $(P>0.05)$

Baseline is first reading immediately post anesthesia

Hypothesis (3): Significant difference between respiratory monitoring parameters with phase one post anesthesia $(30,60,90,120 \mathrm{~min})$. 
Table (6): comparison between control and study groups patient according to respiratory assessment immediately post anesthesia phase one (No. 102).

\begin{tabular}{|c|c|c|c|c|c|c|c|c|c|c|c|c|c|c|c|c|c|c|c|c|c|c|c|c|}
\hline \multirow{3}{*}{$\begin{array}{l}\text { Respiratory } \\
\text { Assessment }\end{array}$} & \multicolumn{4}{|c|}{ Baseline } & \multicolumn{5}{|c|}{$30 \mathrm{~min}$} & \multicolumn{5}{|c|}{$60 \mathrm{~min}$} & \multicolumn{5}{|c|}{$90 \mathrm{~min}$} & \multicolumn{5}{|c|}{$120 \mathrm{~min}$} \\
\hline & \multicolumn{2}{|c|}{$\begin{array}{l}\text { Control } \\
(\mathrm{n}=51)\end{array}$} & \multicolumn{2}{|c|}{$\begin{array}{l}\text { Study } \\
(\mathrm{n}=51)\end{array}$} & \multicolumn{2}{|c|}{$\begin{array}{c}\text { Control } \\
(\mathrm{n}=51)\end{array}$} & \multicolumn{2}{|c|}{$\begin{array}{l}\text { Study } \\
(\mathrm{n}=51)\end{array}$} & \multirow{2}{*}{$\begin{array}{c}P . \\
\text { value }\end{array}$} & \multicolumn{2}{|c|}{$\begin{array}{c}\text { Control } \\
(\mathrm{n}=51)\end{array}$} & \multicolumn{2}{|c|}{$\begin{array}{l}\text { Study } \\
(\mathrm{n}=51)\end{array}$} & \multirow{2}{*}{$\begin{array}{c}P . \\
\text { value }\end{array}$} & \multicolumn{2}{|c|}{$\begin{array}{l}\text { Control } \\
(\mathrm{n}=51)\end{array}$} & \multicolumn{2}{|c|}{$\begin{array}{l}\text { Study } \\
(\mathrm{n}=51)\end{array}$} & \multirow{2}{*}{$\begin{array}{c}P . \\
\text { value }\end{array}$} & \multicolumn{2}{|c|}{$\begin{array}{l}\text { Control } \\
(\mathrm{n}=51)\end{array}$} & \multicolumn{2}{|c|}{$\begin{array}{l}\text { Study } \\
(\mathrm{n}=51)\end{array}$} & \multirow{2}{*}{$\begin{array}{c}\text { P. } \\
\text { value }\end{array}$} \\
\hline & No & $\%$ & No & $\%$ & No & $\%$ & No & $\%$ & & $\begin{array}{l}N \\
0\end{array}$ & $\%$ & $\begin{array}{l}\mathbf{N} \\
0\end{array}$ & $\%$ & & No & $\%$ & No & $\%$ & & No & $\%$ & No & $\%$ & \\
\hline \multicolumn{25}{|l|}{ oxygen mode } \\
\hline 1. Air room & 51 & 100 & 0 & 0.0 & 51 & 100 & 0 & 0.0 & \multirow{2}{*}{$\begin{array}{c}0.000 * \\
*\end{array}$} & 51 & 100 & 0 & 0.0 & \multirow{2}{*}{$0.000^{* *}$} & 51 & 100 & 0 & 0.0 & \multirow{2}{*}{$0.000^{* *}$} & 51 & 100 & 0 & 0.0 & \multirow{2}{*}{$\begin{array}{c}0.000^{*} \\
*\end{array}$} \\
\hline 2.Simple Mask & 0 & 0.0 & 51 & 100 & 0 & 0.0 & 51 & 100 & & 0 & 0.0 & 51 & 100 & & 0 & 0.0 & 51 & 100 & & 0 & 0.0 & 51 & 100 & \\
\hline Fio2 Mean+SD & \multicolumn{2}{|c|}{ Air room } & \multicolumn{2}{|c|}{$40 \pm 0$} & \multicolumn{2}{|c|}{ Air room } & \multicolumn{2}{|c|}{$40 \pm 0$} & NA & \multicolumn{2}{|c|}{ Air room } & \multicolumn{2}{|c|}{$40 \pm 0$} & NA & \multicolumn{2}{|c|}{ Air room } & 40 & & NA & Air & oom & & \pm 0 & $\mathrm{NA}$ \\
\hline $\begin{array}{c}\text { Respiratory rate } \\
\text { Mean } \pm \text { SD }\end{array}$ & 24. & +5.9 & & \pm 5.4 & & +5.7 & 19.6 & \pm 2.6 & $\begin{array}{c}0.001 * \\
*\end{array}$ & & $3+5.5$ & & $8 \pm 1.6$ & $0.001^{* *}$ & $22 \pm$ & -5.3 & 17.8 & & $0.001 * *$ & 21. & \pm 4.7 & 17. & \pm 1.7 & $\begin{array}{c}0.001 * \\
*\end{array}$ \\
\hline $\begin{array}{l}\text { Saturation } \\
\text { Mean+SD }\end{array}$ & 91. & \pm 5.1 & & \pm 3.7 & & $8 \pm 6$ & 97.6 & \pm 12.6 & $\begin{array}{c}0.001 * \\
*\end{array}$ & & $8+4.1$ & & $7 \pm 0.6$ & 0.001 ** & 92.5 & \pm 3.5 & 99.6 & +0.7 & $0.001 * *$ & & & & \pm 0.3 & $\begin{array}{c}0.001^{*} \\
*\end{array}$ \\
\hline
\end{tabular}

Chi-square test was used to compare percentages and independent t-test was used to compare means

* There is a significant difference ( $p<0.05)$

${ }^{N s}$ : There is no significant difference $(p>0.05)$

The baseline is first reading immediately post anesthesia.

Table (1): shows distribution of control group and study group according to their Socio-demographic characteristics (gender, sex), type and duration of anesthesia. The mean + SD age was $(31.5+11.8)$ in the control group and $(31.9+9.2)$ in the study group. It was noticed that $(72.5 \%)$ were male while $(27.5 \%)$ of the patient were female in the control group and $(86.3 \%)$ of patients were male and $(13.7 \%)$ of patients were female in the study group. There were no significant differences between two groups regarding age, sex. It was in both groups (control and study) were having a high percentage, in general, anesthesia $(88.2 \%$ and $90.2 \%)$ respectively, the

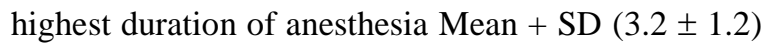
for the control group, compared to study group Mean $+\mathrm{SD}(2.8 \pm 1.0)$. .

Table (2): shows a comparison of the control group and study group according to their Richmond Agitation Sedation Scale (RASS). It was found that the highest percentage in both groups (control and study) for drowsy $(35.3 \%)$ and $(31.4 \%)$ in the first 30 minutes while in the 60 minutes was found highest for alert and calm (98\%) in the study group, compare to control group (72.5\%). Regarding 90 minutes, it was found that highest percentage for alert and calm $(88.2 \%$ and $100 \%)$ in control and study group respectively.

Table (3): shows a comparison of the control group and study group according to their post-operative
** There is a significant difference $(p<0.01)$

${ }^{N A}$ : Not applicable.

complications. It was found that the majority of both control and study group were having high percentage of cardiovascular hypotension and tachycardia $(23.5 \%, 29.4 \%$ vs $15.7 \%, 19.6 \%)$ respectively, in respiratory hypoxia and laryngeal spasm $(15.7 \%$, $17.6 \%$ vs $17.6 \%, 7.8 \%$ ) respectively, in gastric nausea and vomiting similar $(23.5 \%$ vs $15.6 \%)$, respectively, pain related operation and hypothermia $(60.8 \%, 37.3 \%$ vs $56.9 \%, 31.4 \%)$ respectively. There were significant differences between two groups relation to airway obstruction, pain related operation and hypothermia.

Table (4): shows a comparison between both study and control groups in relation to hemodynamic parameters immediately post anesthesia. As regard temperature it was founded that mean value of the study group $(36.9 \pm 0.3)$ while control group was $(35.8 \pm 0.6)$ in 30 minutes with significant difference between both groups at all times. Regarding systolic blood pressure and diastolic blood pressure, there was no significant difference in the first 30 minutes $(\mathrm{P}<$ $0.066 \mathrm{Vs} \mathrm{P}<0.064)$ respectively but show a significant difference in other times. About mean arterial blood pressure there was a significant difference between both groups in $(30,60,90,120)$ minutes. Regarding heart rate show significant difference between study and control groups ( $\mathrm{P}<$ 0.001 ) at 30 minutes, revealed significant decrease in pulse in study group compare with control group in other times. About capillary refill there was a significant difference $(\mathrm{P}<0.001)$ between both 
groups within the same level of difference at all times. Regarding of ECG monitoring, there was a significant difference $(\mathrm{P}<0.001)$ between study group comparing with control group within the same level of difference at all times.

Table (5): represents the comparison between controls and study groups according to face pain scale immediately post anesthesia. It noticed that the Mean \pm SD in control group and study group (5.8 \pm $2.6 \mathrm{Vs} 1.2 \pm 1.1)$ respectively at 30 minutes and there were Mean \pm SD in the other times $(60,90,120)$ minutes founded for control group and study group $(5.3 \pm 2.5 \mathrm{Vs} 0.2 \pm 0.5$ and $5.2 \pm 2.4 \mathrm{Vs} 0.1 \pm 0.5$ and $5 \pm 2.3 \mathrm{Vs} 0 \pm 0$ ) respectively There were significant differences $(\mathrm{p}<0.001)$ between two groups regarding to pain level at all times.

Table (6): show a comparison between both control and study groups in relation to respiratory assessment immediately post anesthesia. It noticed that percentage of oxygen mode in control group (100\%) air room comparing with study group (100\%) simple mask with highly significant difference $(\mathrm{P}<0.000)$ at all times . also show of Mean \pm SD for respiratory rate in control group and study group $(24 \pm 5.7 \mathrm{Vs}$ $19.6 \pm 2.6)$ respectively at 30 minutes and there were Mean \pm SD in the other times $(60,90,120)$ minutes founded for control group and study group (22.3 \pm $5.5 \mathrm{Vs} 17.8 \pm 1.6,22 \pm 5.3 \mathrm{Vs} 17.8 \pm 1.4$ and $21.6 \pm$ $4.7 \mathrm{Vs} 17.3 \pm 1.7)$ respectively and there were significant differences $(\mathrm{P}<0.001)$ between two groups regarding to respiratory rate at all times. About of saturation show significant difference $(\mathrm{P}<$ $0.001)$ between two groups at all times with Mean \pm SD in all times $(30,60,90,120)$ minutes founded for control group and study group $(90.8 \pm 6 \mathrm{Vs} 97.6 \pm$ $12.6,92.8 \pm 4.1 \mathrm{Vs} 99.7 \pm 0.6,92.5 \pm 3.5 \mathrm{Vs} 99.6 \pm$ 0.7 and $93.1 \pm 3.7$ Vs $99.9 \pm 0.3$ ) respectively.

Table (6): (Cont.) It was found that the highest percentage in depth for control and study groups $(47.1 \% \mathrm{Vs} 92.2 \%)$ respectively with significant difference $(\mathrm{P}<0.000)$ in $(30)$ minutes and also in $(60$, 90) minutes show a significant difference between both groups. Regarding pattern of breathing show highest percentage in normal for control and study groups $(58.8 \%$ Vs $94.1 \%)$ respectively, with significant difference $(\mathrm{P}<0.000)$ in $(30)$ minutes. Regarding breathing sound show the highest percentage in normal for control and study groups (68.6 \% Vs 100\%) respectively with significant difference $(\mathrm{P}<0.000)$ in $(30)$ minutes between control and study groups. According to accessory muscles was found that highest percentage not used for both groups $(72.5 \%$ Vs $100.0 \%)$ respectively, with significant difference $(\mathrm{P}<0.000)$ in $(30)$ minutes between control and study groups.

\section{Discussion}

Care in the post anesthesia phase I unit center on providing post anesthesia nursing care and transitioning the patient to the intensive care setting. The post anesthesia care unit nurse is responsible for taking a patient's vital signs following surgery, including blood pressure, respirations, lung sounds and pulse. The focus on the post anesthesia care of the patient with the goals of improving postanesthetic safety and quality of life, reducing postoperative adverse event, providing a uniform assessment of recovery and streamlining postoperative care and discharge criteria. The nurse acts as a patient advocate during this time when the patients are unable to assume responsibility for themselves ( Nagelhout \& Plaus, 2014. Dyer et al., 2008, Smedley \& Goldworthy, 2013 ).

The current study aims to investigate the effect of nursing care guidelines for postanesthetic (phase one) on the patient outcome at Assiut University hospital. Our study showed statistically significance difference of hemodynamic parameters, respiratory status outcomes, decrease pain level and complication. The present study shows socio-demographic data between control and study groups these data reflected the patient with control and study groups in microsurgical reconstructive post anesthesia care unit. The result of the present study revealed that percentage related age in the control group was more than half of patient between 18-30 years old while in the study group was half of patients between 1830years old and there was no statistically significant difference between both groups of the study. The result of our study comes in contrast with (Saleh, 2004), who mentioned age group 21-30 years old was less than one-third of patients in studies groups and also similar with (Iteke, et al., 2011) who reported no statistically significant difference between both groups related age group.

As regard the gender, the current study revealed that two third of the control group was male while more than two third of the study group was male patient with no statistically significant difference between control and study groups. This in line with the study done by (El-khadrawe et al., 2012) who documented two third of the study group were males patients and another study was done by (Iteke et al., 2011) who mentioned two third of the study group were males 
patients with no statistically significant difference between control and study groups.

As regard types of anesthesia, the findings of the present study revealed that majority of the control group and study group were general anesthesia with no statistically significant difference between both groups of the study. This agrees with ( Dijk Van et al., 2012) who reported the majority of general anesthesia in both groups. This finding was supported by the result of (Tennant et al., 2012) who documented that majority of general anesthesia in studies groups.

Regarding duration of anesthesia, the current study showed more than half of patients in control and study groups were the duration of anesthesia between (2-4) hours with on statistically significant difference between both groups of the study. The result of the current study was agreed by (Fleischut et al., 2014) who mentioned the most common of duration group was $(2.0 \sim 2.5)$ hours with the patient has total knee arthroplasty.

As regard Richmond Agitation Sedation Scale, based on the findings of current study mentioned that one third was drowsy level in both groups in the first (30) minutes while alert and calm level highly percentage in $(60,90,120)$ minutes in both groups with statistically significant difference between control and study groups in all interval times except in the (120) minutes no statistically significant difference between control and study groups. This result comes in agreement with (Benítez-Rosario et al., 2013) who reported that majority of patients post anesthesia were alert and calm while highly percentage of patients drowsy and light sedation in both groups.

Our result revealed the most common complication immediately post anesthesia phase one time in control and study groups as cardiovascular were hypotension and tachycardia, respiratory were hypoxia and laryngeal spasm, gastric intestinal were nausea and vomiting and nerves system were pain related operation and hypothermia.

Regarding post anesthesia complication, the most complication of cardiovascular was hypotension and tachycardia in both groups with no statistically significant difference between control and study groups. These disagree with studies were done by (Tennant et al., 2012) who reported about (5\%) cardiovascular complications and also,(Puig-
Barberà et al., 2006) who documented (9.6\%) of cardiovascular complications.

The respiratory complication in the present study was airway obstruction in the control group and no incidence in a study group that improved by use jaw thrust, head tilt, oropharyngeal, or nasopharyngeal airway to maintain an airway with statistically significant difference between control and study groups. In this line for the study group, (Mendonça et al., 2014) who documented that no incidence of airway obstruction complication post anesthesia with intervention in the study group. This is similar to (Visvanathan et al., 2005) reported that airway obstruction complication post anesthesia participated directly to laryngeal spasm and desaturation. The laryngeal spasm and hypoxia in the present study were less than one fifth in control and study groups with no statistically significant difference between control and study groups. These agree with (Visvanathan et al., 2005) reported that Laryngospasm may present atypically and if not promptly managed effectively, may lead to morbidity and mortality of laryngeal spasm. Also, disagree with (Mendonça et al., 2014) who documented that more than tenth of patients post anesthesia presented mildmoderate hypoxia in studies groups.

(Tennant et al., 2012) reported that post anesthesia complications were one-third of the patient have nausea and less than one-third of the patient have to vomit .This is similar with the current study complication of vomiting and disagrees with the current study in nausea that shows with no statistically significant difference between control and study groups.

The result of current study showed the most common of complications of nerves system was pain related operation and hypothermia with improved in study group compare with control group because assessing pain continuously, administer pain medication as prescribed, keep in comfortable position and use warming devices, administer warm IV solution and also showed statistically significant difference between control and study groups. (Interven et al., 2009) who reported some complication presented statistically significant difference with performance nursing intervention; this is similar to the current result. This comes in contrast with a study was done by (Couceiro et al., 2009) that reported incidence postoperative pain was less than of half patients have 
pain. But (Lin et al., 2013) in this line with our result that documented statistically significant difference between control and study groups. The present study disagrees with (Kiekkas et al., 2005) that documented hypothermia complication was showed tow third of patients in both groups.

The study result revealed that there was an improvement in patient hemodynamic parameters (temperature, mean arterial blood pressure, pulse, capillary refill and ECG monitoring) in the entire study time interval post anesthesia of implementation of the nursing care guideline on the study group compared with the control group.

The result of the present study revealed that most patients low body temperature immediately post anesthesia in control group but improved by the nursing care guideline intervention such as (use warming devices, administer warm IV solution and continuously check temperature) in study group with shows highly statistically significant difference between control and study groups through the entire time interval. In this line, (Lin et al., 2013) that reported statistically significant difference between control group and study group. Also, this is similar to (Siddiqui et al., 2013) who reported that body temperature within normal value for post anesthesia patients. This finding similar with (Benson et al., 2012) who said at each of the four post anesthesia care unit assessment time intervals (on admission, 30 minutes after admission, 60 Minutes after admission, and at discharge), mean oral temperatures were significantly higher in the warming gown group than in the standard blanket group $(\mathrm{P}=0.009)$. (Yang et al. 2012)agree with our study who reported that significant difference $(\mathrm{P}<0.001)$ between two groups when used two methods for rewarming.

As regarding monitoring blood pressure immediately post anesthesia in both groups, the current study revealed that mean arterial blood pressure through the entire time interval for control group within normal range but improved and stabilization with intervention in a study group with the highly statistically significant difference between both groups during phase one time. That agrees with studies were done by (Iwata et al., 2008) who reported mean arterial blood pressure for patient post anesthesia in the normal range. (Klimathianaki et al., 2010) who documented who reported mean arterial blood pressure in normal range also supported the present result in the study group.

The present study showed stabilization of heart rate by nursing care guideline intervention for study group compare with a control group with statistically significant difference between control group and study group for heart rate in the post anesthesia phase one all time interval. That agrees with a study done by (Ogawa et al., 2006) who mentioned heart rate within a normal range in the study group. Also, that was in the same line with (Iwata et al., 2008) who reported heart rate post anesthesia normal and stable of post anesthesia patients.

As regard to immediately post anesthesia pain related operation the result of the current study revealed decrease post-operative pain level with continuously evaluated pain level and intervention in study group compare with a control group and there was statistically significant difference between control group and study group at all time intervals. That was in this line (Gilligan 2011) who reported high-level postoperative pain without intervention.

The study result revealed that there was an improvement in patient respiratory status (oxygen mode supplementation, respiratory rate, and oxygen saturation) in all time interval post anesthesia of implementation of the nursing care guideline on the study group compared with the control group.

Based on the findings of oxygen modes immediately post anesthesia revealed control group of oxygen supplementation by air room mode and in a study group with intervention the oxygen supplementation by simple mask mode with flow $3 \mathrm{~L} / \mathrm{hr}$. So, showed highly statistically significance difference between control and study groups for oxygen supplementation and also inspired oxygen fraction $\left(\mathrm{FiO}_{2}\right)$ concentration oxygen supplementation for control and study groups (21\% vs 40\%) respectively. These agree with (Rincón Valenzuela \& Caro, 2012) and (Hovaguimian 2013) who documented high inspired oxygen fraction $\left(\mathrm{FiO}_{2}\right)$ levels reduce post-operative nausea and vomiting and to promote postoperative atelectasis, in this same clinical setting, the risk of surgical site infection and mortality are also reduced. The finding of the current study related to respiratory rate immediately post anesthesia revealed improved and stabilized with intervention in the study group and there were highly statistically significance differences between control and study groups at post- 
anesthetic phase one all time interval. These result supported by (Klimathianaki et al., 2010) who mentioned postoperative the respiratory rate within normal range. Also, the current study supported (Siddiqui et al., 2013) who reported that patients presented with desaturation the respiratory rate was less than normal range post anesthesia.

As regard to oxygen saturation immediately post anesthesia revealed improved with simple mask mode oxygen supplementation and others nursing intervention (patient head position and sectioning) in the study group and there were highly statistically significance differences between control and study groups at post-anesthetic phase one all time interval. This is similar to (Aytac et al., 2014) that who documented post anesthesia saturation within normal. Also (Loick et al., 2006) that supported the current result who reported there was a close relationship between the pre-operative and post-operative incidence of apnea, pre-operative and postoperative desaturation periods, pre-operative and post-operative mean oxygen saturation values, pre-operative and post-operative minimal oxygen saturation values in both groups. The present study not on the line with (Dalchow et al., 2013) that who mentioned saturation less than normal without nursing intervention.

Regarding to depth, pattern breathing, breath sound and accessory muscles immediately post anesthesia the result of the current study revealed that statistically significance differences between control and study groups in $(30,60,90)$ minutes for depth breathing and accessory muscles but showed no statistical significance differences between control and study groups in (120) minutes, also statistically significance differences between control and study groups in the first (30) minutes for pattern breathing, breath sound but no statistically significance difference in $(60,90,120)$ minutes. This is on the line with (Mendonça et al., 2014) who reported inability to deep breath percentage (20.4\%).

Finally, in the fact that study documented the statement of the role of a post anesthesia care unit nurse important vital role in improve post anesthesia care outcomes for the patient and return the patient to the optimal level of physiological functioning. Postanesthetic patients must be monitored and assessed closely for any deterioration in condition and the relevant post anesthesia care plan or pathway must be implemented.

\section{Conclusion}

Based on the finding of the current study, can be concluded that of nursing care guidelines for postanesthetic (phase one) were better than of habitual care of the hospital for microsurgical reconstructive post anesthesia care unit with statistically significant differences between control and study groups. Furthermore, a statistically significant difference were finding between two groups related the patient immediately post anesthesia hemodynamic parameters (temperature, mean arterial blood pressure, pulse, capillary refill and ECG monitoring), respiratory status (oxygen mode supplementation, respiratory rate, and oxygen saturation) that improved with nursing care guidelines intervention in the study group. As regard to pain level statistically significant decrease difference $(p<0.001)$ between control and study groups that nursing guideline assists in decreasing of pain level. As regard to post anesthesia complication reduce with statistically significant difference relation to airway obstruction, pain related operation and hypothermia $(\mathrm{p}<0.009, \quad \mathrm{p}=0.029$ and $\mathrm{p}=0.017)$ respectively between control and study groups.

\section{Recommendations}

From these study findings, it can be recommended the following:

Recommendation regarding education and nursing practice

- Immediately post anesthesia care must be given by training and professional nurses.

- All specialist post anesthesia care unit staff should be appropriately trained, ideally to a nationally recognized nursing guideline.

Recommendation regarding administration

- Should be available post anesthesia care unit with essential equipment for every operation room.

- Provide the nurse with a poster of post anesthesia nursing care guidelines for every post anesthesia care unit.

\section{Recommendation regarding the research}

- Reapply this research on a large probability sample acquired from different geographical areas for generalization.

- Study for assessment of post anesthesia cares nurse's knowledge and practice relation to post anesthesia care. 


\section{Reference}

1. Aytac, I., Postaci, A,. Aytac, B., Sacan, O., Alay, G., Celik, B., Kahveci \& KDikmen, B., (2014): Survey of postoperative residual curarization, acute respiratory events and approach of anesthesiologists. Brazilian Journal of Anesthesiology (English Edition), 1(1-8). Available at: http://linkinghub.elsevier.com

2. Benítez-Rosario, M,. Castillo-Padrós, M., Garrido-Bernet, B., \& González-Guillermo, T., Martínez-Castillo, L., González, A., (2013): Appropriateness and reliability testing of the modified Richmond Agitation-Sedation Scale in Spanish patients with advanced cancer. Journal of pain and symptom management, 45(6), pp.1112-9. Available at: http://www.ncbi.nlm.nih.gov/pubmed/ [Accessed December 26, 2014].

3. Benson, E., McMillan, D. \& Ong, B., (2012): The effects of active warming on patient temperature and pain after total knee arthroplasty. The American journal of nursing, 112(5), pp.26-33; quiz 34, 42. Available at: http://www.ncbi.nlm.nih.gov/pubmed

4. Couceiro, T., Valença, Marcelo M., Lima, L,. De Menezes, T., \& Raposo, M., (2009): Prevalence and Influence of Gender, Age, and Type of Surgery on Postoperative Pain. Brazilian Journal of Anesthesiology, 59(3), pp.314-320. Available at: http://linkinghub.elsevier.com

5. Dalchow, S., Lubeigt, O., Peters, G., Harvey, A., Duggan, T., \& Binning, A., (2013): Transcutaneous carbon dioxide levels and oxygen saturation following caesarean section performed under spinal anaesthesia with intrathecal opioids. International journal of obstetric anesthesia, 22(3), pp.217-22. Available at: http://www.ncbi.nlm.nih.gov/pubmed

6. Davis, M., et al., (2012): Anesthetic management and outcome in patients during endovascular therapy for acute stroke. Anesthesiology, 116(2), pp.396-405. Available at: http://www.ncbi.nlm.nih.gov/pubmed/

7. Dijk Van, J., van Wijck, A., Kappen, T,. Peelen, L., Kalkman, C., \& Schuurmans, M., (2012): Postoperative pain assessment based on numeric ratings is not the same for patients and professionals: a cross-sectional study.
International journal of nursing studies, 49(1), pp.65-71. Available at http://www.ncbi.nlm.nih.gov/pubmed

8. Digiulio, M., Jackson, D., \& Keogh, J., (2007): Medical-Surgical Nursing Demystified, America: The McGraw-Hill Companies. Available at: george_hoare@mcgraw-hill.com.

9. Dyer, R., Piercy, J., Reed, A., Lombard, C., (2008): Hemodynamic Changes Associated with Spinal Anesthesia for Cesarean Delivery in Severe Preeclampsia. American Society of Anesthesiologists, 108(5), pp.802-811. Available at: www.ncbi.nlm.nih.gov/pubmed.

10. El Beheiry, H., \& Mak, P., (2013): Effects of aging and propofol on the cardiovascular component of the autonomic nervous system. Journal of clinical anesthesia, 25(8), pp.637-43. Available at: http://www.ncbi.nlm.nih.gov/pubmed/

11. El-khadrawe, T., Hammad S., \& Hassaan E., (2012): Indicators of outcome after internal fixation of complex acetabular fractures. Alexandria Journal of Medicine, 48(2), pp.99107. Available at: http://linkinghub.elsevier.com

12. Fleischut, P., Eskreis-Winkler, J., GaberBaylis, L., Giambrone, G., Faggiani, S. Dutton, R., \& Memtsoudis, S., (2014): Variability in Anesthetic Care for Total Knee Arthroplasty: An Analysis From the Anesthesia Quality Institute. American journal of medical quality: the official journal of the American College of Medical Quality, 1(1), pp.1-8. Available at: http://www.ncbi.nlm.nih.gov/pubmed

13. Gilligan, M., (2011): Impact of Nursing Interventions on Perioperative Throughput: A Review of The Patient Undergoing Laparoscopic Cholecystectomy In The Ambulatory Surgery Setting Versus Acute Care Setting. ProQuest LLC, 1(1), pp.20-42. Available at: pqdtopen.proquest.com/pqdtopen

14. Hovaguimian, F., (2013): Effect of Intraoperative High Inspired Oxygen Fraction. anaesthesiologists.org, 119(2), pp.303-16. Available at: http//www.archiveouverte.unige.ch/unige.

15. Interven, A., Enfermeiro, E., Complica, A., Em, E., Recupera, S., Las, S., \& Del, I., Complicaciones, L., La, E., Recuperaci, S., 
Popov, C., Peniche, G., (2009): Nurse interventions and the complications in the postanesthesia recovery room. Acta anaesthesiologica Taiwanica: official journal of the Taiwan Society of Anesthesiologists, 43(4), pp.946-52. Available at: www.ee.usp.br/reeusp/

16. Iteke, O., Bakare, M., Agomoh, A., Uwakwe, R. \& Onwukwe, J. 2011. Road traffic accidents and posttraumatic stress disorder in an orthopedic setting in South-Eastern Nigeria: a controlled study. Scandinavian journal of trauma, resuscitation and emergency medicine, 19, p.39. Available at: http://www.pubmedcentral.nih.gov

17. Iwata, M., Inoue, S., Kawaguchi, M., Takahama, M., Tojo, T., Taniguchi, S. \& Furuya, H., (2008): Jugular bulb venous oxygen saturation during one-lung ventilation under sevoflurane- or propofol-based anesthesia for lung surgery. Journal of cardiothoracic and vascular anesthesia, 22(1), pp.71-6. Available at: http://www.ncbi.nlm.nih.gov/pubmed

18. Kiekkas, P., Pouiopoulou, M., Papahatzi, A., \& Souleles, P., (2005): Effects Of Hypothermia And Shivering Onstandard Pacu Monitoring Of Patients. AANA journal/, 73(1), pp.47-52. Available at: www.aana.com/members/journal

19. Klimathianaki, M., Kondili, E., Alexopoulou, C., Prinianakis, G., \& Georgopoulos, D., (2010): Effect of propofol on breathing stability in adult ICU patients with brain damage. Respiratory physiology \& neurobiology, 171(3), pp.232-8. Available at: http://www.ncbi.nlm.nih.gov/pubmed[Accessed December 26, 2014]

20. Ledowski, T., Reimer, M., Chavez, V., Kapoor, V., \& Wenk, M., (2012): Effects of acute postoperative pain on catecholamine plasma levels, hemodynamic parameters, and cardiac autonomic control. Pain, 153(4), pp.75964. Available at: http://www.ncbi.nlm.nih.gov/pubmed[Accessed December 9, 2014]

21. Lethbridge, L., (2008): A literature review: nursing assessment in a post anaesthetic care unit. American journal of surgery, 10(6), pp.2528. Available at: http://journals.sfu.ca [Accessed October 27, 2014]

22. Lin, H., Ting, P., Chang, W., Yang, M., Chang, C., \& Chou, A., (2013): Predictive risk index and prognosis of postoperative reintubation after planned extubation during general anesthesia: a single-center retrospective case-controlled study in Taiwan from 2005 to 2009. Acta anaesthesiologica Taiwanica : official journal of the Taiwan Society of Anesthesiologists, 51(1), pp.3-9. Available at: http://www.ncbi.nlm.nih.gov/pubmed

23. Loick, H.m Schwann, G., Radig-Thomas, H., \& Theissen, J., (2006): The effect of general anaesthesia on breathing patterns in elderly patients during the early post-operative period. European Journal of Anaesthesiology, 14(3), pp.258-265. Available at: http://journals.cambridge.org

24. Mendonça, J., Pereira, H., Xará, D., Santos, A., \& Abelha, F., (2014): Obese patients: Respiratory complications in the post-anesthesia care unit. Revista Portuguesa de Pneumologia (English Edition), 20(1), pp.12-19. Available at: http://linkinghub.elsevier.com

25. Nagelhout, J., \& Plaus, K., (2014): Nurse anesthesia 5th ed., America: Saunders,an imperint of Elsevier Inc. Available at: www.elsevier.com

26. Ogawa, Y., Iwasaki, K ., Shibata, S., Kato, J., Ogawa, S., \& Oi, Y., (2006): Different effects on circulatory control during volatile induction and maintenance of anesthesia and total intravenous anesthesia: autonomic nervous activity and arterial cardiac baroreflex function evaluated by blood pressure and heart rate variability a. Journal of clinical anesthesia, 18(2), pp.87-95. Available at: http://www.ncbi.nlm.nih.gov/pubmed

27. Oliveira, R., Tenório, S., Tanaka, P., \& Precoma, D., (2012): Control of pain through epidural block and incidence of cardiac dysrhythmias in postoperative period of thoracic and major abdominal surgical procedures: a comparative study. Revista brasileira de anestesiologia, 62(1), pp.10-8. Available at: http://www.ncbi.nlm.nih.gov/pubmed

28. Preston, N., \& Gregory, M., (2012): Patient recovery and post- anaesthesia care unit (PACU). Anaesthesia and Intensive Care Medicine, 13(12), pp.591-593. Available at: http://dx.doi.org. 
29. Pudner, R., (2010): Nursing the Surgical Patient 3rd ed., London: Elsevier Ltd. Available at: www.elseverhealth.com

30. Puig-Barberà, J., Márquez-Calderón, S., \& Vila-Sánchez, M., (2006): Cardiac Complications of Major Elective Non-Cardiac Surgery: Incidence and Risk Factors. Revista Española de Cardiología (English Edition), 59(4), pp.329-337. Available at: http://linkinghub.elsevier.com

31. Rabadi, D., (2013): Effect of normal saline administration on circulation stability during general anesthesia induction with propofol in gynecological procedures - randomisedcontrolled study. Revista brasileira de anestesiologia, 63(3), pp.258-61. Available at: http://www.ncbi.nlm.nih.gov/pubmed

32. Rincón Valenzuela, D., \& Caro, A., (2012): Use of intra-operative supplemental oxygen to reduce morbidity and mortality in general anesthesia: systematic review and meta-analysis of randomized controlled trials. Colombian Journal of Anesthesiology, 40(1), pp.34-51. Available at: http://linkinghub.elsevier.com

33. Saleh, W., (2004): Tendon Transfer around the Shoulder Joint for Late Case Obstetrical Brachial Plexus Injury. Assiut University

34. Sessler, C., Gosnell, M., \& Grap, M., (2003): Richmond Agitation Sedation Scale (RASS). Jama, 166(2), p.2991. Available at: http://www.sedaicu.

35. Siddiqui, N., Arzola, C., Teresi, J., \& Guerina, L., (2013): Predictors of desaturation in the postoperative anesthesia care unit: an observational study 5 . Journal of Clinical Anesthesia, 25(1), pp.612-617. Available at: www.elsevier.com

36. Silverstein, J., Barlow, J., Chung, F., Connis, R., \& Hunt, S., (2013): Practice Guidelines for Postanesthetic Care. American Society of Anesthesiologists, 5(2). Available at: www.anesthesiology.org

37. Smedley, P., \& Goldworthy, S., (2013): British Anaesthetic and Recovery Nurses Association [BARNA] Standards of Practice. British Anesthetic and Rcovery Nursing Association, (October), pp.5-23. Available at: www.barna.co.uk
38. Tennant, I., Augier, R., Crawford-Sykes, A., Ferron-Boothe, D., Gordon-Strachan, G., \& Harding-Goldson, H., (2012): Minor postoperative complications related to anesthesia in elective gynecological and orthopedic surgical patients at a teaching hospital in Kingston, Jamaica. Revista brasileira de anestesiologia, 62(2), pp.188-98. Available at: http://www.ncbi.nlm.nih.gov/pubmed

39. Timby, B., \& Smith, N., (2010): Introductory Medical-Surgical Nursing 10th ed., Philadelphia: Wolters Kluwer Health | Lippincott Williams \& Wilkins. Available at: WWW.LWW.COM

40. Visvanathan, T., Kluger, M., Webb, R., \& Westhorpe, R., (2005): Crisis management during anaesthesia: laryngospasm. Quality \& safety in health care, 14(3), p.e3. Available at:

41. an Beest, P., et al., (2014): Tissue oxygenation as a target for goal-directed therapy in high-risk surgery: a pilot study. BMC anesthesiology, 14, p.122. Available at: http://www.pubmedcentral.nih.govhttp://www.p ubmedcentral.nih.gov

42. Warden V., Hurley A., \& Volicer L., (2011): Faces Pain Scale Very Hurts just a little bit Hurts a little more Hurts even more Hurts a whole lot Hurts as much as you can imagine Visual Analog Scale (VAS ) Pain Assessment of Infants Journal of the American Medical Directors Association. Journal of the American Medical Directors Association, pp.1-4. Available at: www.ttuhsc.edu/provost/clinic

43. Yang, H., et al., (2012): The comparison of two recovery room warming methods for hypothermia patients who had undergone spinal surgery. Journal of nursing scholarship: an official publication of Sigma Theta Tau International Honor Society of Nursing / Sigma Theta Tau, 44(1), pp.2-10. Available at: http://www.ncbi.nlm.nih.gov/pubmed/ 